$11-1-2013$

\title{
Distribution of the Ratio of Normal and Rice Random Variables
}

Nayereh B. Khoolenjani

University of Isfahan, Isfahan, Iran, n.b.khoolenjani@gmail.com

Kavoos Khorshidian

Shiraz University, Shiraz, Iran

Follow this and additional works at: http://digitalcommons.wayne.edu/jmasm

Part of the Applied Statistics Commons, Social and Behavioral Sciences Commons, and the Statistical Theory Commons

\section{Recommended Citation}

Khoolenjani, Nayereh B. and Khorshidian, Kavoos (2013) "Distribution of the Ratio of Normal and Rice Random Variables," Journal of Modern Applied Statistical Methods: Vol. 12 : Iss. 2 , Article 27.

DOI: $10.22237 /$ jmasm/1383279960

Available at: http://digitalcommons.wayne.edu/jmasm/vol12/iss2/27

This Emerging Scholar is brought to you for free and open access by the Open Access Journals at DigitalCommons@WayneState. It has been accepted for inclusion in Journal of Modern Applied Statistical Methods by an authorized editor of DigitalCommons@WayneState. 


\section{Emerging Scholars: Distribution of the Ratio of Normal and Rice Random Variables}

\author{
Nayereh B. Khoolenjani \\ University of Isfahan \\ Isfahan, Iran
}

\author{
Kavoos Khorshidian \\ Shiraz University \\ Shiraz, Iran
}

The ratio of independent random variables arises in many applied problems. The distribution of the ratio $|X / Y|$ is studied when $X$ and $Y$ are independent Normal and Rice random variables, respectively. Ratios of such random variables have extensive applications in the analysis of noises in communication systems. The exact forms of probability density function (PDF), cumulative distribution function (CDF) and the existing moments are derived in terms of several special functions. As a special case, the PDF and CDF of the ratio of independent standard Normal and Rayleigh random variables have been obtained. Tabulations of associated percentage points and a computer program for generating tabulations are also given.

Keywords: Normal distribution, Rice distribution, ratio random variable, special functions.

\section{Introduction}

For given random variables $X$ and $Y$, the distribution of the ratio $|X / Y|$ arises in a wide range of natural phenomena of interest, such as in engineering, hydrology, medicine, number theory, psychology, etc. More specifically, Mendelian inheritance ratios in genetics, mass to energy ratios in nuclear physics, target to control precipitation in meteorology, inventory ratios in economics are exactly of this type. The distribution of the ratio random variables (RRV) has been extensively investigated by many authors especially when $X$ and $Y$ are independent and belong to the same family. Various methods have been compared and reviewed by authors including Pearson (1910), Greay (1930), Marsaglia (1965, 2006) and Nadarajah (2006).

Nayereh B. Khoolenjani is a Ph.D. student in the Department of Statistics. Email at: n.b.khoolenjani@gmail.com. Kavoos Khorshidian is in the Department of Statistics. 


\section{KHOOLENJANI \& KHORSHIDIAN}

The exact distribution of $|X / Y|$ is derived when $X$ and $Y$ are independent random variables (RVs) having Normal and Rice distributions with parameters $\left(\mu, \sigma^{2}\right)$ and $(\lambda, v)$, respectively. The Normal and Rice distributions are well known and of common use in engineering, especially in signal processing and communication theory. In engineering, there are many real situations where measurements could be modeled by Normal and Rice distributions. Some typical situations in which the ratio of Normal and Rice random variables appear are as follows. In the case that $X$ and $Y$ represent the random noises corresponding to two signals, studying the distribution of the quotient $|X / Y|$ is of interest. For example in communication theory it may represent the relative strength of two different signals and in MRI, it may represent the quality of images. Moreover, because of the important concept of moments of RVs as magnitude of power and energy in physical and engineering sciences, the possible moments of the ratio of Normal and Rice random variables have been also obtained. Applications of Normal and Rice distributions and the ratio RVs may be found in Rice (1974), Helstrom (1997), Karagiannidis and Kotsopoulos (2001), Salo, et al. (2006), Withers and Nadarajah (2008) and references therein.

The probability density function (PDF) of a two-parameter Normal random variable $X$ can be written as:

$$
f_{X}(x)=\frac{1}{\sqrt{2 \pi} \sigma} \exp \left\{-\frac{1}{2 \sigma^{2}}(x-\mu)^{2}\right\},-\infty<x<\infty
$$

where $-\infty<\mu<\infty$ is the location parameter and $\sigma>0$ is the scale parameter. For $\mu=0$ and $\sigma^{2}=1$, (1) becomes the distribution of standard Normal random variable. A well known representation for CDF of $X$ is as

$$
F_{X}(x)=\frac{1}{2}\left\{1+\operatorname{erf}\left(\frac{x-\mu}{\sigma \sqrt{2}}\right)\right\}
$$

where $\operatorname{erf}(\cdot)$ denotes the error function that is given by

$$
\operatorname{erf}(x)=\frac{2}{\sqrt{\pi}} \int_{0}^{x} e^{-u^{2}} d u
$$


Also,

$$
E\left(X^{k}\right)=\mu^{k} \cdot k ! \sum_{j=0}^{\left[\frac{k}{2}\right]} \frac{1}{(k-2 j) ! j !}\left(\frac{\sigma^{2}}{2 \mu^{2}}\right)^{j}
$$

If $Y$ has a Rice distribution with parameters $(\lambda, v)$, then the PDF of $Y$ is as follows:

$$
f_{Y}(y)=\frac{y}{\lambda^{2}} \exp \left\{-\frac{\left(y^{2}+v^{2}\right)}{2 \lambda^{2}}\right\} \mathrm{I}_{0}\left(\frac{y v}{\lambda^{2}}\right), y>0, v \geq 0, \lambda>0
$$

where $y$ is the signal amplitude, $\mathrm{I}_{0}($.$) is the modified Bessel function of the first$ kind of order $0,2 \lambda^{2}$ is the average fading-scatter component and $v^{2}$ is the lineof-sight (LOS) power component. The Local Mean Power is defined as $\Omega=2 \lambda^{2}+v^{2}$ which equals $E\left(X^{2}\right)$, and the Rice factor $K$ of the envelope is defined as the ratio of the signal power to the scattered power, i.e., $K=v^{2} / 2 \lambda^{2}$. When $K$ goes to zero, the channel statistic follows Rayleigh distribution, whereas if $K$ goes to infinity, the channel becomes a non-fading channel. For $v=0$, the expression (5) reduces to a Rayleigh distribution.

\section{Notations and Preliminaries}

Recall some special mathematical functions, these will be used repeatedly throughout this study. The modified Bessel function of first kind of order $v$, is

$$
I_{v}(x)=\left(\frac{1}{2} x\right)^{v} \sum_{k=0}^{\infty} \frac{\left(\frac{1}{4} x^{2}\right)^{k}}{(k !) \Gamma(v+k+1)}
$$

The generalized hypergeometric function is denoted by

$$
{ }_{p} F_{q}\left(a_{1}, a_{2}, \ldots, a_{p} ; b_{1}, b_{2}, \ldots, b_{q} ; z\right)=\sum_{k=0}^{\infty} \frac{\left(a_{1}\right)_{k}\left(a_{2}\right)_{k} \ldots\left(a_{p}\right)_{k}}{\left(b_{1}\right)_{k}\left(b_{2}\right)_{k} \ldots\left(b_{q}\right)_{k}} \frac{z^{k}}{k !}
$$




\section{KHOOLENJANI \& KHORSHIDIAN}

The Gauss hypergeometric function and the Kummer confluent hypergeometric function are given, respectively, by

$$
{ }_{2} F_{1}(a, b ; c ; z)=\sum_{k=0}^{\infty} \frac{(a)_{k}(b)_{k}}{(c)_{k}} \frac{z^{k}}{k !}
$$

and

$$
{ }_{1} F_{1}(a ; b ; z)=\sum_{k=0}^{\infty} \frac{(a)_{k} z^{k}}{(b)_{k} k !}
$$

where $(a)_{k},(b)_{k}$ represent Pochhammer's symbol given by

$$
(a)_{k}=a(a+1) \cdots(a+k-1)=\frac{\Gamma(\alpha+k)}{\Gamma(\alpha)} \text {. }
$$

The parabolic cylinder function is

$$
D_{v}(z)=2^{\frac{v}{2}} e^{-\frac{z^{2}}{4}} \Psi\left(-\frac{1}{2} v, \frac{1}{2} ; \frac{1}{2} z^{2}\right)
$$

where $\Psi(a, c ; z)$ represents the confluent hypergeometric function given by

$$
\Psi(a, c ; z)=\Gamma\left[\begin{array}{l}
1-c \\
1+a-c
\end{array}\right]{ }_{1} F_{1}(a ; c ; z)+\Gamma\left[\begin{array}{l}
c-1 \\
a
\end{array}\right] 2^{1-c}{ }_{1} F_{1}(1+a-c ; 2-c ; z)
$$

in which

$\Gamma\left[\begin{array}{l}a_{1}, \ldots, a_{m} \\ b_{1}, \ldots, b_{n}\end{array}\right]=\frac{\prod_{i=1}^{m} \Gamma\left(a_{i}\right)}{\prod_{j=1}^{n} \Gamma\left(b_{j}\right)}$.

The complementary error function is denoted by 
DISTRIBUTION RATIO OF NORMAL AND RICE RANDOM VARIABLES

$$
\operatorname{erfc}(x)=\frac{2}{\sqrt{\pi}} \int_{x}^{\infty} e^{-u^{2}} d u
$$

The following lemmas are of frequent use.

Lemma 1 (Equation (2.15.5.4), Prudnikov, et al., 1986). For $\operatorname{Re} p>0$, $\operatorname{Re}(\alpha+v)>0 ;|\arg c|<\pi$

$$
\begin{aligned}
& \int_{0}^{\infty} x^{\alpha-1} e^{-p x^{2}} I_{v}(c x) d x \\
& =2^{-v-1} c^{v} p^{-\frac{(\alpha+v)}{2}} \Gamma\left[\begin{array}{c}
\frac{(\alpha+v)}{2} \\
v+1
\end{array}\right]{ }_{1} F_{1}\left(\frac{\alpha+v}{2} ; v+1 ; \frac{c^{2}}{4 p}\right)
\end{aligned}
$$

Lemma 2 (Equation (2.8.9.2), Prudnikov, et al., 1986). For $\operatorname{Re} p>0$; $|\arg c|<\frac{\pi}{4}$

$$
\begin{aligned}
& \int_{0}^{\infty} x^{2 n+1} e^{-p x^{2}}\left\{\begin{array}{l}
\operatorname{erf}(c x+b) \\
\operatorname{erfc}(c x+b
\end{array}\right\} d x=\left\{\begin{array}{l}
0 \\
1
\end{array}\right\} \frac{n !}{2 p^{n+1}} \pm \frac{(-1)^{n}}{2} \\
& \frac{\partial^{n}}{\partial P^{n}}\left[\frac{1}{p} \operatorname{erf}(b)+\frac{c}{p \sqrt{c^{2}+p}} \exp \left(-\frac{p b^{2}}{c^{2}+p}\right) \operatorname{erf}\left(\frac{b c}{\sqrt{c^{2}+p}}\right)\right] .
\end{aligned}
$$

Lemma 3 (Equation (3.462.1), Gradshteyn \& Ryzhik, 2000). For $\operatorname{Re} \beta>0$, $\operatorname{Re} v>0$

$$
\int_{0}^{\infty} x^{v-1} \exp \left(\left\{-\beta x^{2}-\gamma x\right\} d x=(2 \beta)^{-\frac{v}{2}} \Gamma(v) \exp \left(\frac{\gamma^{2}}{8 \beta}\right) D_{-v}\left(\frac{\gamma}{\sqrt{2 \beta}}\right) .\right.
$$




\section{KHOOLENJANI \& KHORSHIDIAN}

\section{The Ratio of Normal and Rice Random Variables}

The explicit expressions for the PDF and CDF of $|X / Y|$ are derived in terms of the Gauss hypergeometric function. The ratio of standard Normal and Rayleigh $\mathrm{RVs}$ is also considered as a special case.

Theorem 1: Suppose that $X$ and $Y$ are independent Normal and Rice random variables with parameters $\left(\mu, \sigma^{2}\right)$ and $(\lambda, v)$, respectively. The PDF of the ratio random variable $T=|X / Y|$ can be expressed as $f(t)=g(t)+g(-t)$, where

$$
\begin{aligned}
g(t)= & \frac{e^{-\left\{\frac{v^{2}}{2 \lambda^{2}}+\frac{\mu^{2}}{2 \sigma^{2}}-\frac{\mu^{2} t^{2} \lambda^{2}}{4 \sigma^{2}\left(\lambda^{2} t^{2}+\sigma^{2}\right)} \sigma^{2} \lambda\right.}}{\sqrt{2 \pi}\left(\lambda^{2} t^{2}+\sigma^{2}\right)^{\frac{3}{2}}} \\
& \times \sum_{k=0}^{\infty} \frac{\left(\frac{v^{2}}{4 \lambda^{2}}\right)^{k}}{(k !)^{2}} \cdot \Gamma(2 k+3) \cdot D_{-(2 k+3)}\left(\frac{-\mu t \lambda}{\sigma \sqrt{\lambda^{2} t^{2}+\sigma^{2}}}\right) .
\end{aligned}
$$

\section{Theorem 1 Proof:}

$$
\begin{aligned}
f(t) & =\int_{0}^{\infty} y f_{X}(t y) f_{Y}(y) d y+\int_{0}^{\infty} y f_{X}(-t y) f_{Y}(y) d y \\
& =\int_{0}^{\infty} y \cdot \frac{1}{\sqrt{2 \pi} \sigma} \exp \left\{-\frac{1}{2 \sigma^{2}}(t y-\mu)^{2}\right\} \cdot \frac{y}{\lambda^{2}} \exp \left\{-\frac{\left(y^{2}+v^{2}\right)}{2 \lambda^{2}}\right\} I_{0}\left(\frac{y v}{\lambda^{2}}\right) d y \\
& +\int_{0}^{\infty} y \cdot \frac{1}{\sqrt{2 \pi} \sigma} \exp \left\{-\frac{1}{2 \sigma^{2}}(-t y-\mu)^{2}\right\} \cdot \frac{y}{\lambda^{2}} \exp \left\{-\frac{\left(y^{2}+v^{2}\right)}{2 \lambda^{2}}\right\} I_{0}\left(\frac{y v}{\lambda^{2}}\right) d y
\end{aligned}
$$

The two integrals in (13) can be calculated by direct application of Lemma 3. Thus the result follows.

Remark 2: By using expression (10), elementary forms for $g(t)$ in Theorem 1 can be derived as follows: 
DISTRIBUTION RATIO OF NORMAL AND RICE RANDOM VARIABLES

$$
g(t)=\frac{e^{-\frac{1}{2 \sigma^{2} \lambda^{2}\left(v^{2} \sigma^{2}+\mu^{2} \lambda^{2}\right)} \lambda \sigma^{2}}}{\sqrt{2 \pi}\left(t^{2} \lambda^{2}+\sigma^{2}\right)^{\frac{3}{2}}} \sum_{k=0}^{\infty} \frac{\left(\frac{v^{2}}{4 \lambda^{2}}\right)^{k} \Gamma(2 k+3)}{(k !)^{2} 2^{\frac{2 k+3}{2}}} \Psi\left(\frac{2 k+3}{2}, \frac{1}{2} ; \frac{\mu^{2} t^{2} \lambda^{2}}{2 \sigma^{2}\left(t^{2} \lambda^{2}+\sigma^{2}\right)}\right)
$$

Corollary 3 Assume that $X$ and $Y$ are independent standard Normal and Rayleigh random variables, respectively. The PDF of the ratio random variable $T=|X / Y|$ can be expressed as

$$
f_{T}(t)=\frac{\lambda}{\left(t^{2} \lambda^{2}+1\right)^{3 / 2}} \quad, t>0
$$

Theorem 4: Suppose that $X$ and $Y$ are independent Normal and Rice random variables with parameters $\left(\mu, \sigma^{2}\right)$ and $(\lambda, v)$, respectively. The CDF of the ratio random variable $T=|X / Y|$ can be expressed as $F(t)=G(t)-G(-t)$ where

$$
\begin{aligned}
G(t) & =\frac{e^{-\frac{v^{2}}{2 \lambda^{2}}}}{2 \lambda^{2}} \sum_{k=0}^{\infty} \frac{\left(\frac{v^{2}}{4 \lambda^{4}}\right)^{k}}{(k !)^{2}}\left\{\frac{n !}{2\left(\frac{1}{2 \lambda^{2}}\right)^{k+1}}-\frac{(-1)^{k}}{2} \frac{\partial^{k}}{\partial\left(\frac{1}{2 \lambda^{2}}\right)^{k}}\left[2 \lambda^{2} \operatorname{erf}\left(\frac{-\mu}{\sqrt{2} \sigma}\right)\right.\right. \\
& \left.\left.-\frac{2 t \lambda^{3}}{\sqrt{t^{2} \lambda^{2}+\sigma^{2}}} \times \exp \left(-\frac{\mu^{2}}{2\left(t^{2} \lambda^{2}+\sigma^{2}\right)}\right) \operatorname{erfc}\left(-\frac{\mu t \lambda}{\sigma \sqrt{2\left(t^{2} \lambda^{2}+\sigma^{2}\right)}}\right)\right]\right\} .
\end{aligned}
$$

Theorem 4 Proof: The CDF $F(t)=\operatorname{Pr}(|X / Y| \leq t)$ can be written as

$$
F(t)=\int_{0}^{\infty}\left\{\Phi\left(\frac{t y-\mu}{\sigma}\right)-\Phi\left(\frac{-t y-\mu}{\sigma}\right)\right\} f_{Y}(y) d y
$$

where $\Phi($.$) is the cdf of the standard Normal distribution. Using the relationship$

$$
\Phi(-x)=\frac{1}{2} \operatorname{erfc}\left(\frac{x}{\sqrt{2}}\right)
$$

Eq. (17) can be rewritten as 


\section{KHOOLENJANI \& KHORSHIDIAN}

$$
\begin{aligned}
F(t)= & \frac{1}{2} \int_{0}^{\infty}\left\{\operatorname{erfc}\left(\frac{\mu-t y}{\sigma \sqrt{2}}\right)-\operatorname{erfc}\left(\frac{\mu+t y}{\sigma \sqrt{2}}\right)\right\} f_{Y}(y) d y \\
= & \frac{1}{2} \int_{0}^{\infty} \operatorname{erfc}\left(\frac{\mu-t y}{\sigma \sqrt{2}}\right) \cdot \frac{y}{\lambda^{2}} \exp \left\{-\frac{\left(y^{2}+v^{2}\right)}{2 \lambda^{2}}\right\} I_{0}\left(\frac{y v}{\lambda^{2}}\right) d y \\
& -\frac{1}{2} \int_{0}^{\infty} \operatorname{erfc}\left(\frac{\mu+t y}{\sigma \sqrt{2}}\right) \cdot \frac{y}{\lambda^{2}} \exp \left\{-\frac{\left(y^{2}+v^{2}\right)}{2 \lambda^{2}}\right\} I_{0}\left(\frac{y v}{\lambda^{2}}\right) d y .
\end{aligned}
$$

The result follows by using Lemma 2 .

Corollary 5: Assume that $X$ and $Y$ are independent Normal and Rice random variables with parameters $\left(0, \sigma^{2}\right)$ and $(\lambda, 0)$, respectively. The CDF of the ratio random variable $T=|X / Y|$ is

$$
F(t)=\frac{t \lambda}{\sqrt{t^{2} \lambda^{2}+\sigma^{2}}}, \quad t>0 .
$$

Figures (1) and (2) illustrate possible shapes of the pdf corresponding to (20) for different values of $\sigma^{2}$ and $\lambda$. Note that the shape of the distribution is mainly controlled by the values of $\sigma^{2}$ and $\lambda$.

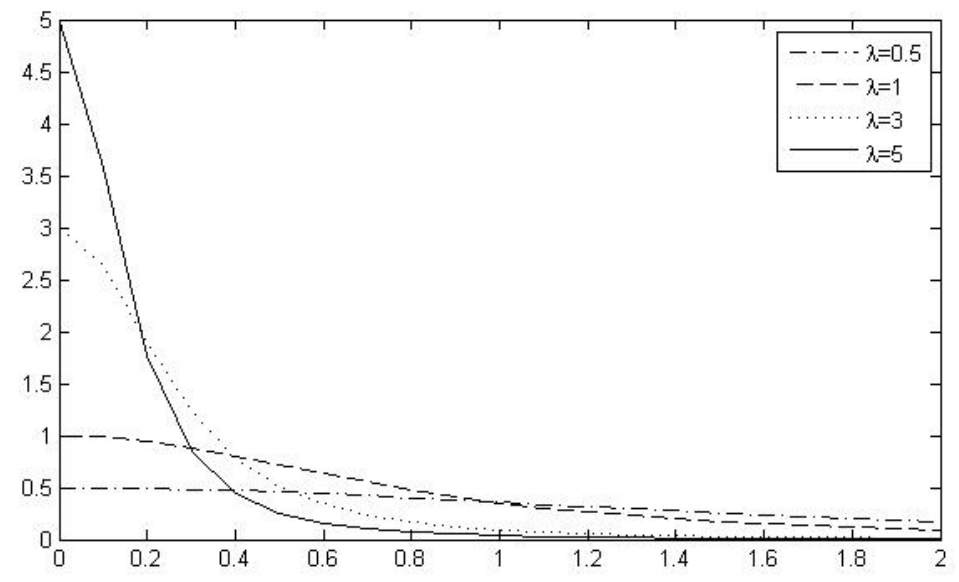

Figure 1 Plots of the pdf corresponding to (20) for $\lambda=0.5,1,3,5$ and $\sigma=1$. 


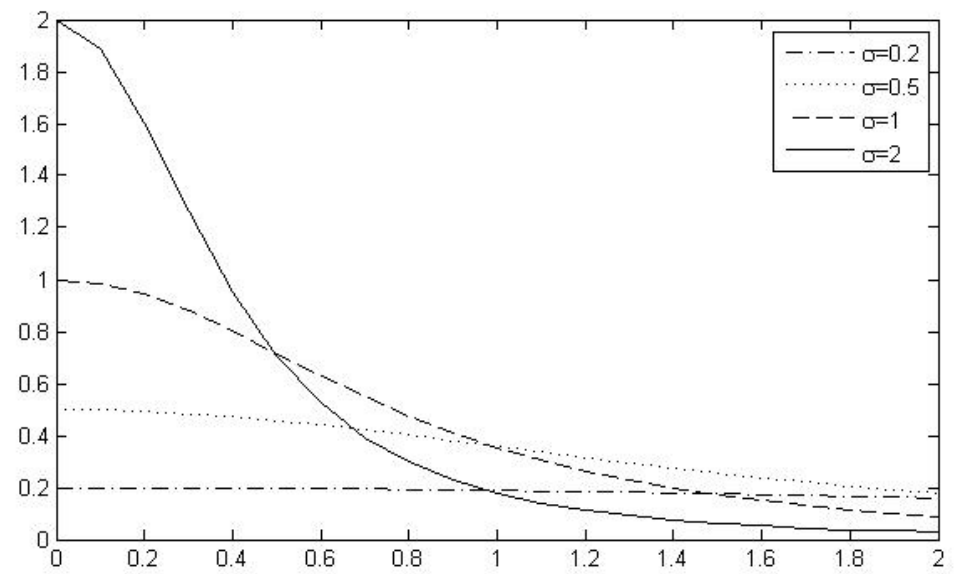

Figure 2 Plots of the pdf corresponding to (20) for $\sigma=0.2,0.5,1,2$ and $\lambda=1$.

\section{$\boldsymbol{K}^{\text {th }}$ Moments of the Ratio Random Variable}

In the sequel, the independence of $X$ and $Y$ are used several times for computing the moments of the ratio random variable. The results obtained are expressed in terms of confluent hypergeometric functions.

Theorem 6: Suppose that $X$ and $Y$ are independent Normal and Rice random variables with parameters $\left(\mu, \sigma^{2}\right)$ and $(\lambda, v)$, respectively. A representation for the $\mathrm{k}^{\text {th }}$ moment of the ratio random variable $T=X / Y$, for $k<2$, is:

$$
E\left[T^{k}\right]=\left(\frac{\mu}{\sqrt{2} \lambda}\right)^{k} k ! e^{-\frac{v^{2}}{2 \lambda^{2}}} \Gamma\left(\frac{-k+2}{2}\right)_{1} F_{1}\left(\frac{-k+2}{2} ; 1 ; \frac{v^{2}}{2 \lambda^{2}}\right) \sum_{j=0}^{\left[\frac{k}{2}\right]} \frac{1}{(k-2 j) ! j !}\left(\frac{\sigma^{2}}{2 \mu^{2}}\right)^{j}
$$

Theorem 6 Proof: Using the independency of $X$ and $Y$ the expected ratio can be written as

$$
E\left(T^{k}\right)=E\left(\frac{X^{k}}{Y^{k}}\right)=E\left(X^{k}\right) E\left(\frac{1}{Y^{k}}\right),
$$

in which 


\section{KHOOLENJANI \& KHORSHIDIAN}

$$
E\left(\frac{1}{Y^{k}}\right)=\int_{0}^{\infty} \frac{1}{y^{k}} \cdot \frac{y}{\lambda^{2}} \exp \left\{-\frac{\left(y^{2}+v^{2}\right)}{2 \lambda^{2}}\right\} I_{0}\left(\frac{y v}{\lambda^{2}}\right) d y
$$

By using lemma 2.1, the integral (23) reduces to

$$
E\left(\frac{1}{Y^{k}}\right)=\frac{e^{-\frac{v^{2}}{2 \lambda^{2}}}}{\left(2 \lambda^{2}\right)^{\frac{k}{2}}} \Gamma\left(\frac{-k+2}{2}\right)_{1} F_{1}\left(\frac{-k+2}{2} ; 1 ; \frac{v^{2}}{2 \lambda^{2}}\right)
$$

The desired result now follows by multiplying (4) and (24).

Remark 7: Formula (21), displays the exact forms for calculating $E(T)$, which have been expressed in terms of confluent hypergeometric functions. The deltamethod can be used to approximate the first and second moments of the ratio $T=X / Y$. In detail, by taking $\mu_{X}=E(X), \mu_{Y}=E(Y)$ and using the Deltamethod (Casella \& Berger, 2002) results in:

$$
E(T) \approx \frac{\mu_{X}}{\mu_{Y}}=\sqrt{\frac{2}{\pi}} \frac{\mu e^{\frac{v^{2}}{2 \lambda^{2}}}}{\lambda_{1} F_{1}\left(\frac{3}{2}, 1 ; \frac{v^{2}}{2 \lambda^{2}}\right)} .
$$

For approximating $\operatorname{Var}(T)$, first recall that $E\left(X^{2}\right)=\mu^{2}+\sigma^{2}$ and $E\left(Y^{2}\right)=2 \lambda^{2}+v^{2}$. Thus,

$\operatorname{Var}\left(\frac{X}{Y}\right) \approx\left(\frac{\mu_{X}^{2}}{\mu_{Y}^{2}}\right)\left(\frac{\operatorname{Var}(X)}{\mu_{X}^{2}}+\frac{\operatorname{Var}(Y)}{\mu_{Y}^{2}}\right)$,

which involves confluent hypergeometric functions, but in simpler forms.

Remark 8: The numerical computation of the obtained results in this article entails calculation of special functions, their sums and integrals, which have been tabulated and available in determinds books and computer algebra packages (see Greay, 1930; Helstrom, 1997; and Salo, et al. 2006 for more details. 


\section{DISTRIBUTION RATIO OF NORMAL AND RICE RANDOM VARIABLES}

\section{Percentiles}

Table 1. Percentage points of $T=|X / Y|$ for $\lambda=0.1-2.5$.

\begin{tabular}{|c|c|c|c|c|c|c|}
\hline$\lambda$ & $p=0.01$ & $p=0.05$ & $p=0.1$ & $p=0.9$ & $p=0.95$ & $p=0.99$ \\
\hline 0.1 & 0.100005 & 0.500626 & 1.005023 & 20.64741 & 30.4243 & 70.1792 \\
\hline 0.2 & 0.050002 & 0.250313 & 0.502518 & 10.32370 & 15.2121 & 35.0896 \\
\hline 0.3 & 0.033335 & 0.166875 & 0.335012 & 6.882471 & 10.1414 & 23.3930 \\
\hline 0.4 & 0.025001 & 0.125156 & 0.251259 & 5.16185 & 7.6060 & 17.5448 \\
\hline 0.5 & 0.020001 & 0.100125 & 0.201007 & 4.12948 & 6.0848 & 14.0358 \\
\hline 0.6 & 0.016667 & 0.083437 & 0.167506 & 3.44123 & 5.0707 & 11.6965 \\
\hline 0.7 & 0.014286 & 0.071518 & 0.143576 & 2.94963 & 4.3463 & 10.0256 \\
\hline 0.8 & 0.012503 & 0.062578 & 0.125629 & 2.58092 & 3.8030 & 8.7724 \\
\hline 0.9 & 0.011111 & 0.055625 & 0.111670 & 2.29415 & 3.3804 & 7.7976 \\
\hline 1 & 0.010002 & 0.050062 & 0.100503 & 2.06474 & 3.0424 & 7.0179 \\
\hline 1.1 & 0.009091 & 0.045511 & 0.091367 & 1.87703 & 2.7658 & 6.3799 \\
\hline 1.2 & 0.008333 & 0.041718 & 0.083753 & 1.72061 & 2.5353 & 5.8482 \\
\hline 1.3 & 0.0076926 & 0.038509 & 0.077310 & 1.58826 & 2.3403 & 5.3984 \\
\hline 1.4 & 0.0071432 & 0.035759 & 0.071788 & 1.47481 & 2.1731 & 5.0128 \\
\hline 1.5 & 0.0066670 & 0.033375 & 0.067002 & 1.37649 & 2.0282 & 4.6786 \\
\hline 1.6 & 0.0062503 & 0.031289 & 0.062814 & 1.29046 & 1.9015 & 4.3862 \\
\hline 1.7 & 0.0058826 & 0.029448 & 0.059119 & 1.21455 & 1.7896 & 4.1281 \\
\hline 1.8 & 0.0055558 & 0.027812 & 0.055835 & 1.14707 & 1.6902 & 3.8988 \\
\hline 1.9 & 0.0052634 & 0.026348 & 0.052896 & 1.08670 & 1.6012 & 3.6936 \\
\hline 2 & 0.0050002 & 0.025031 & 0.050251 & 1.03237 & 1.5212 & 3.5089 \\
\hline 2.1 & 0.0047621 & 0.023839 & 0.047858 & 0.98321 & 1.4487 & 3.3418 \\
\hline 2.2 & 0.0045456 & 0.022755 & 0.045683 & 0.93851 & 1.3829 & 3.1899 \\
\hline 2.3 & 0.0043480 & 0.021766 & 0.043697 & 0.89771 & 1.3227 & 3.0512 \\
\hline 2.4 & 0.0041668 & 0.020859 & 0.041876 & 0.86030 & 1.2676 & 2.9241 \\
\hline 2.5 & 0.0040002 & 0.020025 & 0.040201 & 0.82589 & 1.2169 & 2.8071 \\
\hline
\end{tabular}




\section{KHOOLENJANI \& KHORSHIDIAN}

Table 2. Percentage points of $T=|X / Y|$ for $\lambda=2.6-5$.

\begin{tabular}{|c|c|c|c|c|c|c|}
\hline$\lambda$ & $p=0.01$ & $p=0.05$ & $p=0.1$ & $p=0.9$ & $p=0.95$ & $p=0.99$ \\
\hline 2.6 & 0.0038463 & 0.019254 & 0.038655 & 0.79413 & 1.1701 & 2.6992 \\
\hline 2.7 & 0.0037038 & 0.018541 & 0.037223 & 0.76471 & 1.1268 & 2.5992 \\
\hline 2.8 & 0.0035716 & 0.017879 & 0.035894 & 0.73740 & 1.0865 & 2.5064 \\
\hline 2.9 & 0.0034484 & 0.017262 & 0.034656 & 0.71197 & 1.0491 & 2.4199 \\
\hline 3 & 0.0033335 & 0.016687 & 0.033501 & 0.68824 & 1.0141 & 2.3393 \\
\hline 3.1 & 0.0032259 & 0.016149 & 0.032420 & 0.66604 & 0.9814 & 2.2638 \\
\hline 3.2 & 0.0031251 & 0.015644 & 0.031407 & 0.64523 & 0.9507 & 2.1931 \\
\hline 3.3 & 0.0030304 & 0.015170 & 0.030455 & 0.62567 & 0.9219 & 2.1266 \\
\hline 3.4 & 0.0029413 & 0.014724 & 0.029559 & 0.60727 & 0.8948 & 2.0640 \\
\hline 3.5 & 0.0028572 & 0.014303 & 0.028715 & 0.58992 & 0.8692 & 2.0051 \\
\hline 3.6 & 0.0027779 & 0.013906 & 0.027917 & 0.57353 & 0.8451 & 1.9494 \\
\hline 3.7 & 0.0027028 & 0.013530 & 0.027163 & 0.55803 & 0.8222 & 1.8967 \\
\hline 3.8 & 0.0026317 & 0.013174 & 0.026448 & 0.54335 & 0.8006 & 1.8468 \\
\hline 3.9 & 0.0025642 & 0.012836 & 0.025770 & 0.52942 & 0.7801 & 1.7994 \\
\hline 4 & 0.0025001 & 0.012515 & 0.025125 & 0.51618 & 0.7606 & 1.7544 \\
\hline 4.1 & 0.0024391 & 0.012210 & 0.024513 & 0.50359 & 0.7420 & 1.7116 \\
\hline 4.2 & 0.0023810 & 0.011919 & 0.023929 & 0.4916 & 0.7243 & 1.6709 \\
\hline 4.3 & 0.0023256 & 0.011642 & 0.023372 & 0.48017 & 0.7075 & 1.6320 \\
\hline 4.4 & 0.0022728 & 0.011377 & 0.022841 & 0.46925 & 0.6914 & 1.5949 \\
\hline 4.5 & 0.0022223 & 0.011125 & 0.022334 & 0.45883 & 0.6760 & 1.5595 \\
\hline 4.6 & 0.0021740 & 0.010883 & 0.021848 & 0.44885 & 0.6613 & 1.5256 \\
\hline 4.7 & 0.0021277 & 0.010651 & 0.021383 & 0.43930 & 0.6473 & 1.4931 \\
\hline 4.8 & 0.0021145 & 0.010532 & 0.020672 & 0.42654 & 0.6311 & 1.4752 \\
\hline 4.9 & 0.0021073 & 0.010380 & 0.019823 & 0.41839 & 0.6277 & 1.4613 \\
\hline 5 & 0.0020094 & 0.010157 & 0.018782 & 0.41027 & 0.6120 & 1.4479 \\
\hline
\end{tabular}

Tabulations of percentage points $t_{p}$ associated with the cdf (20) of $T=|X / Y|$ are provided. These values are obtained by numerically solving:

$$
\frac{t_{p} \lambda}{\sqrt{t_{p}{ }^{2} \lambda^{2}+\sigma^{2}}}=p
$$




\section{DISTRIBUTION RATIO OF NORMAL AND RICE RANDOM VARIABLES}

Tables 1 and 2 provide the numerical values of $t_{p}$ for $\lambda=0.1,0.2, \ldots, 5$ and $\sigma=1$. It is hoped that these numbers will be of use to practitioners as mentioned in the introduction. Similar tabulations could be easily derived for other values of $\lambda, \sigma$ and $p$ by using the sample program provided in Appendix A.

\section{References}

Casella, G., \& Berger, L. B. (2002), Statistical Inference. Duxbury Press.

Gradshteyn, I. S., \& Ryzhik, I. M. (2000).Table of Integrals, Series, and Products. San Diego, CA: Academic Press.

Greay R. C. (1930). The frequency distribution of the quotient of two normal variates. Journal of the Royal Statistical Society. 93, 442-446.

Helstrom, C. (1997). Computing the distribution of sums of random sine waves and the Rayleigh-distributed random variables by saddle-point integration. IEEE Trans. Commun. 45(11), 1487-1494.

Karagiannidis, G. K., \& Kotsopoulos, S. A. (2001). On the distribution of the weighted sum of L independent Rician and Nakagami Envelopes in the presence of AWGN. Journal of Communication and Networks. 3(2), 112-119.

Marsaglia, G. (1965). Ratios of Normal Variables and Ratios of Sums of Uniform. JASA. 60, 193, 204.

Marsaglia, G. (2006). Ratios of Normal Variables. Journal of Statistical Software. 16(4), 1-10.

Nadarajah, S. (2006). Quotient of Laplace and Gumbel random variables. Mathematical Problems in Engineering. vol. 2006. Article ID 90598, 7 pages.

Pearson, K. (1910). On the constants of Index-Distributions as Deduced from the Like Constants for the Components of the Ratio with Special Reference to the Opsonic Index. Biometrika. 7(4), 531-541.

Prudnikov, A. P., Brychkov Y.A., Marichev O.I. (1986). Integrals and Series, 2. New York: Gordon and Breach.

Rice, S. (1974). Probability distributions for noise plus several sin waves the problem of computation. IEEE Trans. Commun. 851-853.

Salo, J., EI-Sallabi, H. M., \& Vainikainen, P. (2006). The distribution of the product of independent Rayleigh random variables. IEEE Transactions on Antennas and Propagation. 54(2), 639-943. 


\section{KHOOLENJANI \& KHORSHIDIAN}

Withers, C. S. \& Nadarajah, S. (2008). MGFs for Rayleigh Random Variables. Wireless Personal Communications. 46(4), 463-468.

\section{Appendix A}

The following program in $\mathrm{R}$ can be used to generate tables similar to that presented in the section headed 'Percentiles.'

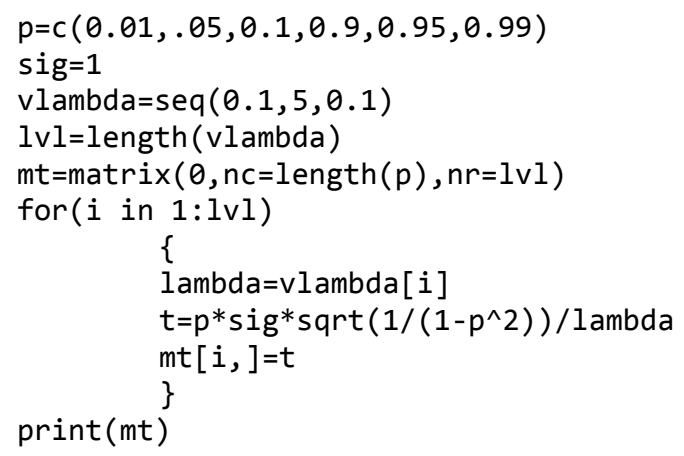

